\title{
New Cs sputter ion source with polyatomic ion beams for secondary ion mass spectrometry applications
}

\author{
S. F. Belykh and V. V. Palitsin \\ Department of Physics, University of Warwick, Warwick Conventry CV4 7AL, United Kingdom \\ I. V. Veryovkin \\ Argonne National Laboratory, Materials Science Division, Argonne, Illinois 60439 \\ A. P. Kovarsky \\ Ioffe Physical-Technical Institute, 194021 St. Petersburg, Russia \\ R. J. H. Chang \\ Department of Physics, University of Warwick, Warwick Conventry CV4 7AL, United Kingdom
}

A. Adriaens

Department of Analytical Chemistry, Ghent University, B-9000 Ghent, Belgium

M. G. Dowsett

Department of Physics, University of Warwick, Warwick Conventry CV4 7AL, United Kingdom

F. Adams

Department of Chemistry, University of Antwerp, 2610 Antwerp, Belgium

(Received 17 April 2007; accepted 27 June 2007; published online 2 August 2007)

A simple design for a cesium sputter ion source compatible with vacuum and ion-optical systems as well as with electronics of the commercially available Cameca IMS-4f instrument is reported. This ion source has been tested with the cluster primary ions of $\mathrm{Si}_{n}{ }^{-}$and $\mathrm{Cu}_{n}{ }^{-}$. Our experiments with surface characterization and depth profiling conducted to date demonstrate improvements of the analytical capabilities of the secondary ion mass spectrometry instrument due to the nonadditive enhancement of secondary ion emission and shorter ion ranges of polyatomic projectiles compared to atomic ones with the same impact energy. (C) 2007 American Institute of Physics.

[DOI: $10.1063 / 1.2761021]$

\section{INTRODUCTION}

Secondary ion mass spectrometry (SIMS) is a widely used analytical technique in research and in development, manufacture, and problem solving for various materials. ${ }^{1-3} \mathrm{~A}$ continuous evolution of the SIMS technique is needed to keep pace with the demands of front-line research and development in these fields, especially regarding the lateral and depth resolution. One of the potential ways to improve the analytical capabilities of existing SIMS instrumentation is to use polyatomic, rather than atomic, ions as projectiles.

The bombardment of solids by small cluster ions with energies of a few $\mathrm{keV} /$ atom leads to (a) a nonadditive enhancement in sputtering ${ }^{4}$ and improved secondary ion yields, ${ }^{5,6}$ and (b) a reduced penetration depth of probe atoms ${ }^{7}$ and a reduction of ion-beam-induced damage. ${ }^{8-10}$ These fundamental features of "cluster-solid" interaction show a possibility to improve the surface specificity and depth resolution of the SIMS analysis and to enhance the elemental and molecular detection sensitivity.

Molecular dynamics simulations ${ }^{11-16}$ indicate that the significant factors underlying the unique sputtering characteristics of polyatomic particles are (1) the reduced depth of the energy deposition in the sample during the sputtering event and (2) the time and space coincident coupling of energy transfer from the collision cascades of the individual atoms in the primary ion to the sputtered molecule. For a polyatomic projectile there is a higher probability that multiple collision cascades are generated simultaneously from the collision of the constituent projectile atoms with the substrate molecules/atoms. There is, therefore, an enhancement in the observed sputter yield due to the "nonadditive" effects of the collision cascades.

Most commercially available SIMS instruments are equipped with ion sources that produce mainly atomic or light diatomic projectiles such as $\mathrm{Cs}^{+}$(surface ionization ion source), $\mathrm{Ga}^{+}$(liquid metal ion source), $\mathrm{Ar}^{+}, \mathrm{Xe}^{+}, \mathrm{O}^{-}$, and $\mathrm{O}_{2}^{+}$ (duoplasmatron ion source). Modifications of these ion sources can also produce a limited set of molecular or cluster ions (see, for example, Refs. 17-21). At present, there is a rising interest and demand in the SIMS community for cluster ion sources for use in organic analysis and, especially, on time-of-flight SIMS instruments (several cluster ion sources aimed at such applications are available, e.g., Refs. 22-25). There also remains a need for improvements in inorganic dynamic SIMS applications, where cluster ions in a beam of moderate current would be of interest. From this perspective, a robust and durable cluster ion source is needed as its development would enable an effective and relatively inexpensive way of enhancing analytical capabilities of SIMS instruments. 
An ion source which utilizes negative ions generated by bombarding a target with $\mathrm{keV} \mathrm{Cs}^{+}$ions is one suitable candidate. The main advantage of such a source is the possibility of producing negative cluster ions from almost all elements. Negative primary ions do not cause as much surface charging as do positive ions, which is important for SIMS analysis with nonconducting or poorly conducting samples.

Various sputter ion source designs have been developed and combined with homemade SIMS instruments. ${ }^{26-32}$ For example, in the ion source design described in Ref. 32, a gold target was sputtered by a $3 \mathrm{keV} \mathrm{Cs}^{+}$beam with a $30 \mu \mathrm{A}$ ion current, which produced emission of a variety of the $\mathrm{Au}_{m}{ }^{-}$ions that after mass separation could be focused into a $\sim 0.5 \mathrm{~mm}$ diameter beam spot with 5-6 nA ion currents for $\mathrm{Au}_{3}{ }^{-}$.

Recently, Gillen et al. ${ }^{33}$ and Gillen and Fahey ${ }^{34}$ have tested the first commercially available sputter ion source design (Peabody Scientific PSX-120, Peabody, MA) on the standard Cameca IMS $3 \mathrm{f}$ instrument. It was shown that the use of cluster $\mathrm{C}_{n}{ }^{-}$and $\mathrm{CsC}_{n}{ }^{-}$ions substantially improves the capabilities of the SIMS instrument for the characterization of organic samples and depth profiling of semiconductor materials. However, authors of Refs. 33 and 34 have concluded that this design has some disadvantages (such as the low efficiency of the ion source, the instability of the cluster ion beam, and the contamination of the primary ion beam column by cesium), which limit its use for the routine analysis.

In the present work, a simple design for a cesium sputter ion source free from the above mentioned disadvantages and compatible with vacuum and ion-optical systems as well as with electronics of the Cameca IMS-4f instrument is reported. This work started in the framework of the NATO Science for Peace Program ${ }^{35}$ at Arifov Institute of Electronics (Uzbekistan) and was then continued first at the University of Antwerp (Belgium) and later at the University of Warwick (UK). The source has been tested with primary atomic and cluster ions $\mathrm{Si}_{n}{ }^{-}$and $\mathrm{Cu}_{n}{ }^{-}(n=1,2)$. Results are presented, demonstrating how the use of cluster projectiles improves instrument capabilities in surface characterization and depth profiling.

\section{DESIGN REQUIREMENTS OF THE CESIUM SPUTTER CLUSTER ION SOURCE}

Our development of the sputter cluster ion source started from the summary by Gillen et al. $^{33}$ and Gillen and Fahey ${ }^{34}$ of the design and disadvantages of the first commercially available ion source. Their experience with this ion source indicated the following: (1) Operating with the $\mathrm{Cs}^{+}$ion current of 1-10 mA, the source consumed substantial electric power and required water cooling for both the sputter target and the source chamber. (2) Due to the space charge effect, the intense $\mathrm{Cs}^{+}$ion beam cannot be well focused on the graphite area of the sputter target. As a result, the sputtered $\mathrm{C}_{n}{ }^{-}$and $\mathrm{C}_{n} \mathrm{Cs}^{-}$ions cannot be focused to the small spot on the sample surface without substantial loss in ion current. (3) Intense $\mathrm{Cs}^{+}$ion bombardment leads to both a time dependent decrease in the extracted cluster ion currents and degradation in ion beam focus quality due to the rapid formation of a crater on the sputter target surface. (4) The cesium vapor filling the ion source chamber contaminates the surfaces of the insulators used in the assembly of the ion source and in the ion optics downstream the primary ion beam column, resulting in electric arcing that causes instabilities in the cluster ion beam, decreases the source lifetime, and requires more frequent cleaning of the primary ion-optical column.

To avoid these drawbacks, the new design of the sputter cluster ion source needs to satisfy the following criteria.

- The design should be simple, robust, and compatible with vacuum, ion-optical, and power supply systems of the SIMS instrument. It should not need water cooling.

- The $\mathrm{Cs}^{+}$ion gun should be long-lasting in operation and low in power consumption. It should not produce excessive cesium vapor in the source chamber.

- The combination "ion source-SIMS instrument" should be able to produce cluster ion beams with parameters (such as type, current and kinetic energy of the cluster ions, the diameter of the ion beam spot on the sample surface, etc.) optimized for different SIMS analyses.

- The shape of the sputter target should assure both the stable cluster ion beam current and the focus on the analyzed sample during a long time sputtering.

To address the above requirements, the operating principle of the $\mathrm{Cs}^{+}$ion gun, the sputter target shape, and the mutual arrangement of both the $\mathrm{Cs}^{+}$ion gun and the target were changed in the sputter ion source design described below.

\section{SPUTTER CLUSTER ION SOURCE DESIGN ADAPTED TO CAMECA IMS SERIES INSTRUMENTS}

This work focuses on the development of a sputter ion source compatible with the Cameca IMS-4f and similar SIMS instruments. The ion source is based on a transmission geometry proposed by Middleton. ${ }^{36}$ In such a configuration, the primary $\mathrm{Cs}^{+}$ions and the sputtered negative ions are coaxial and in the same direction. A schematic drawing of the ion source design is given in Fig. 1(a). This device can be mounted on the Cameca IMS-4f instrument without modification as a replacement for the duoplasmatron ion source. It consists of two vacuum housings: the source chamber and the adaptor to connect the source chamber to the ion column of the SIMS instrument. The $\mathrm{Cs}^{+}$ion gun (1), the extraction electrode (2), and two filaments for heating the container and the ionizer of the $\mathrm{Cs}^{+}$ion gun are mounted in the source chamber. The adaptor houses the shield electrode (3), the sputter cone target $(4)$, and two electrodes $(5,6)$ of the immersion lens. The original extraction electrode (7) of the Cameca duoplasmatron ion source was used as the third electrode of the immersion lens. Separating the vacuum housing of the $\mathrm{Cs}^{+}$ion gun from that of the sputter target allows easy servicing of the gun and replacement of the sputter target.

The $\mathrm{Cs}^{+}$ion gun (1) is based on the low power consumption $\mathrm{Cs}^{+}$ion gun of the IMS-4f instrument, where the ionizer has been modified to increase the ion emission efficiency. ${ }^{37}$ The modified ionizer consists of the dense bunch of tungsten wires with a diameter of $20 \mu \mathrm{m}$ and a length of $3 \mathrm{~mm}$ 

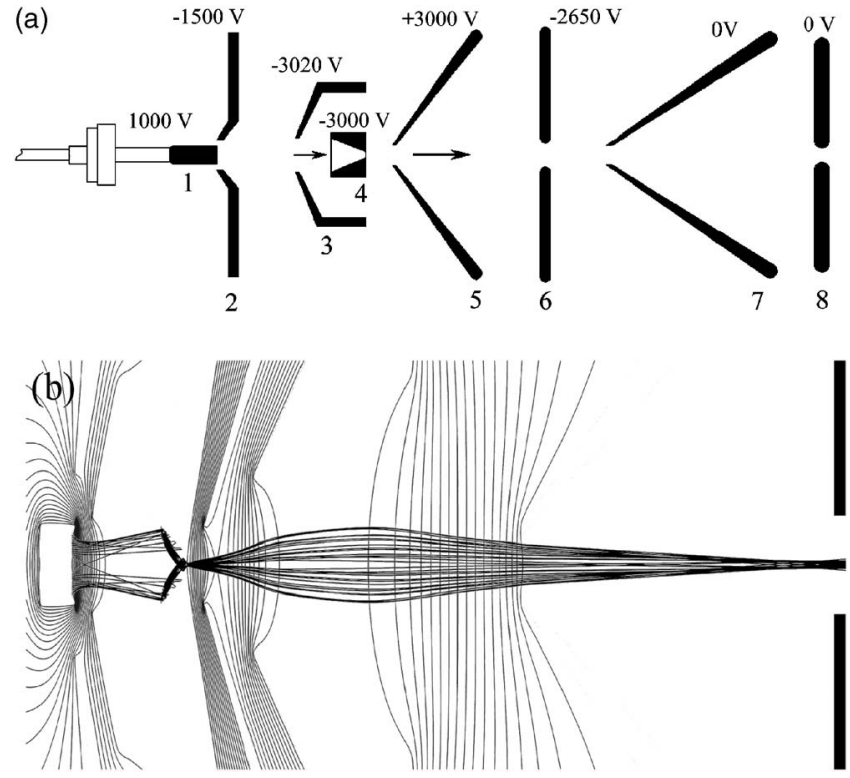

FIG. 1. (a) A schematic diagram of the sputter ion source: 1, the $\mathrm{Cs}^{+}$ion gun; 2 , the extraction electrode; 3 , the shield electrode; 4 , the sputter cone target; 5-7, the three-electrode immersion lens; 8 , the entrance aperture. (b) Computer model of the sputter ion source (in two dimensions) shown with ion trajectories of sputtered negative ions in the "sputter cone targetentrance aperture" space. The model was created in the SIMION 3D $\odot$ computer code Ref. 30

pressed into a tantalum tube with an inner diameter of $3 \mathrm{~mm}$. A tungsten rod with a diameter of $1 \mathrm{~mm}$ has been embedded into the center of the tungsten wire bunch. Sputter targets were machined from $\mathrm{Si}$ and $\mathrm{Cu}$ in the shape of a $6 \mathrm{~mm}$ long rod, with a conical hole drilled through its center (using diamond tools for the silicon). Diameters of the entrance and exit holes of this "hollow cone" target (the "cone" target, in what follows) were 5 and $1 \mathrm{~mm}$, respectively.

The operating principle of the sputter ion source is as follows. Cesium chloride loaded into the container $(\sim 0.1 \mathrm{~g})$ is used as a source material for $\mathrm{Cs}^{+}$ion production. Due to heating of the container and the ionizer by an electron bombardment (the filaments are used as electron sources), molecules of $\mathrm{CsCl}$ evaporate and penetrate the ionizer. On contact with the hot tungsten surface of the ionizer (temperature $\sim 1100{ }^{\circ} \mathrm{C}$ ) they dissociate, and the resulting surfaceionized $\mathrm{Cs}^{+}$ions are accelerated to the cone target by a negative bias. Due to its geometry, the ionizer forms a slightly diverging and hollow $\mathrm{Cs}^{+}$ion beam with a current of 0.1-0.2 mA that sputters the cone target, but does not pass through the exit aperture into the primary ion column. The $\mathrm{Cs}^{+}$ion beam can be directed to different regions of the cone target surface by varying the negative potential applied to the extraction electrode (2). Negative ions sputtered by the $\mathrm{Cs}^{+}$ projectiles are extracted by the "push-pull" electric field, forming a crossover behind the exit aperture. The push-pull ${ }^{38}$ field is created inside the cone space due to the superposition of electric fields applied between "shield electrode-target" and "target-first electrode of the immersion lens." The negatively charged shield electrode (3) prevents the ionizer from damage due to negative ion bombardment. The use of the slightly diverging hollow $\mathrm{Cs}^{+}$ion beam reduces the influence of space charge effects on the crossover formation for negative ions.

Optimum conditions for operating the Cameca IMS-4f SIMS instrument with the original duoplasmatron ion source occur when the image of the crossover is formed near the entrance aperture (8) of the primary ion beam column. To operate the IMS-4f instrument with the sputter ion source, the immersion lens must form an image of the crossover at the same position. However, positions and diameters of both the crossover and its image depend on a set of ion-optical parameters (such as mutual arrangement, dimensions, shapes, and electrical potentials of the electrodes). Therefore, before the new source design was implemented, it was simulated and improved using the SIMION 3D $\odot$ computer code. ${ }^{39}$

Both the ion trajectories simulated when the crossover image is located near the entrance aperture plane and the corresponding ion-optical alignments are shown in Fig. 1(b). The results presented in this figure correspond to the situation where the sample is sputtered by negative ions $\left(\mathrm{Si}_{m}{ }^{-}\right.$and $\mathrm{Cu}_{m}{ }^{-}$) with an energy of $7.5 \mathrm{keV}$ (the nominal potential of the analyzed sample in the Cameca IMS-4f instrument is $+4.5 \mathrm{kV})$.

These simulations show that certain features of the cone target, as a negative ion emitter, must be taken into account for an optimum operation of the sputter ion source. Firstly, the fraction of sputtered ions that makes up the extracted ion beam as well as the position and diameter of the crossover depend on the location of the emission area on the target surface. This is illustrated in Figs. 2(a)-2(c), where the trajectories of ions sputtered from emission areas located near the exit hole, the middle, and the entrance hole of the cone target, respectively, are presented. The emission region near the entrance hole makes a smaller contribution $(\sim 3 \%)$ to the flux of extracted ions and creates a smaller diameter $(\sim 0.17 \mathrm{~mm})$ crossover. Conversely, the emission region near the exit hole makes a larger contribution $(\sim 86 \%)$ into the extracted ion flux and forms a larger diameter $(\sim 0.54 \mathrm{~mm})$ crossover. The optimum lies somewhere between these two extremes [see Fig. 3(b)], where the emission area makes a contribution of $\sim 13 \%$ to the extracted ion flux. In this case, the diameters of the crossover and its image are of 0.22 and $0.51 \mathrm{~mm}$, respectively. Thus, moving the $\mathrm{Cs}^{+}$ion beam along the cone surface can play an important role in the formation of the negative ion beam. Secondly, compared with a conventional planar target where all sputtered species are removed, thus quickly forming a crater on the surface, the ion bombardment of the cone target leads preferentially to the removal of the ions, while the neutrals, which constitute the vast majority of the sputtered flux, redeposit on areas of the target that face the sputtering point. Thus, during the sputtering of the cone surface, the removal of the target material appears to be small, and the sputter deposition prevents a fast formation of a crater on the target surface.

The results of computer simulations were used for designing of the sputter ion source. It was also our intention to minimize alterations/modifications of the IMS- $4 \mathrm{f}$ instrument. Therefore, the device was to be used with the original Cam- 


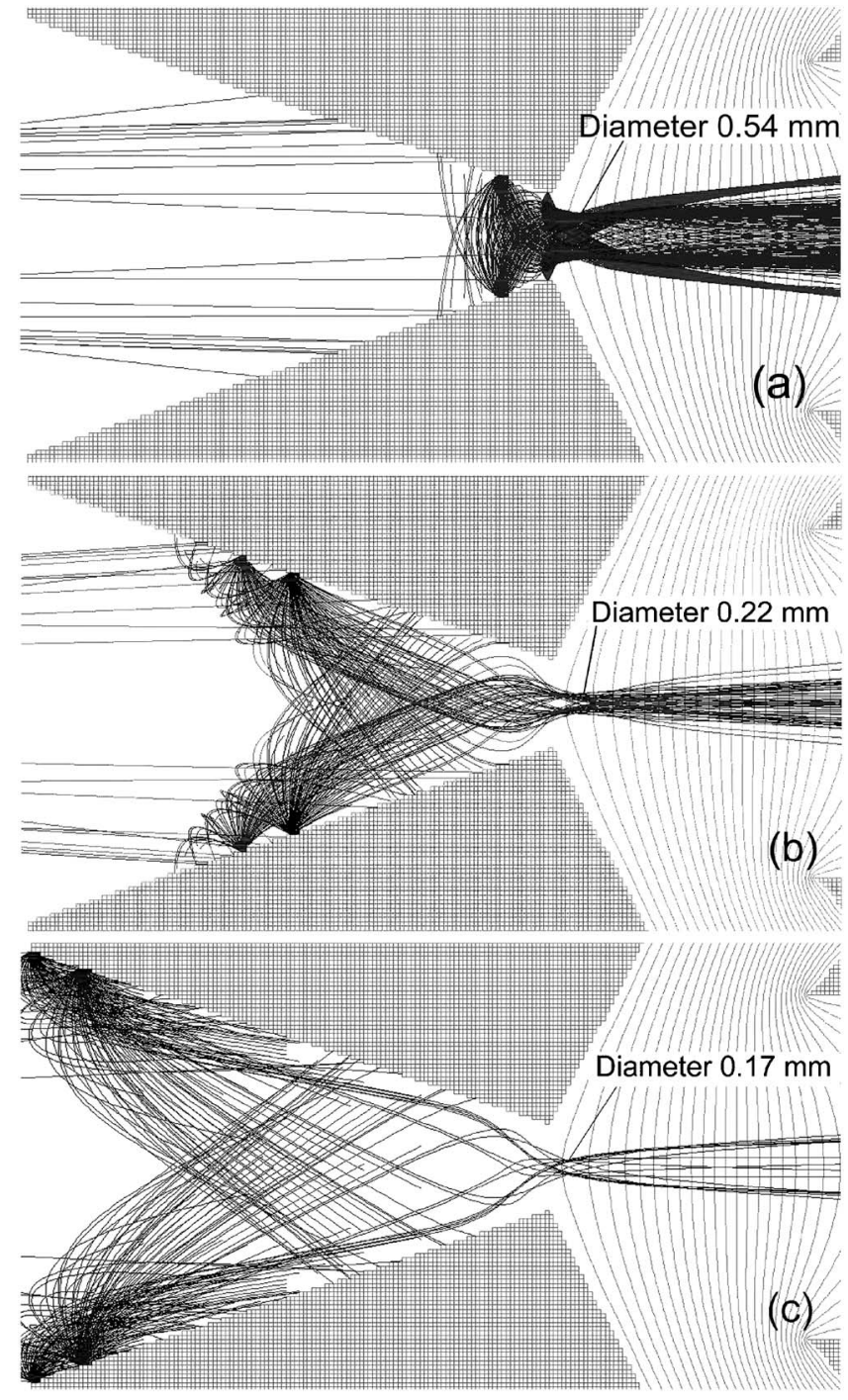

FIG. 2. The ion trajectories of negative ions sputtered from various emission areas located near (a) the exit hole, (b) the middle, and (c)the entrance hole of the "cone" target, respectively.

eca power supplies. Only one additional power supply was needed to set the high potential on the second electrode of the immersion lens.

\section{EXPERIMENTS WITH THE SPUTTER ION SOURCE}

The experiments with surface characterization and depth profiling were carried out with the aim of evaluating the analytical capabilities of the Cameca IMS-4f instrument equipped with the sputter ion source. It is well known that the sputter ion source produces the more intense ion beam when gold is used as a sputter target. However, due to a limited mass range ( $\leqslant 200 \mathrm{amu})$ of the primary mass separator of the IMS-4f instrument, the cluster ions of heavy elements such as $\mathrm{Au}_{m}{ }^{-}$, known also to cause substantially stronger nonadditive enhancement in secondary ion emission, could not be used, and lighter $\mathrm{Si}_{m}{ }^{-}$and $\mathrm{Cu}_{m}{ }^{-}$projectiles were chosen for these studies.

\section{A. Parameters of the cluster ion beams}

The following parameters of the cluster ion beams were achieved.

- Sputtering of the silicon cone target by the $7.5 \mathrm{keV} \mathrm{Cs}^{+}$ ions with an ion current of $\sim 60 \mu \mathrm{A}$ generated the $\mathrm{Si}_{m}{ }^{-}$ cluster ions $(m=1-6)$. After accelerating, focusing, and mass separating operations the typical $\mathrm{Si}_{m}{ }^{-}$ion beam currents measured by the Faraday cup of the SIMS instrument were of 5.6, 2.8, and $0.48 \mathrm{nA}$ for $\mathrm{Si}^{-}, \mathrm{Si}_{2}{ }^{-}$, and $\mathrm{Si}_{3}{ }^{-}$, respectively.

- Sputtering of the copper cone target by the $4.5 \mathrm{keV} \mathrm{Cs}^{+}$ ions with currents of $\sim 150 \mu \mathrm{A}$ produced $\mathrm{Cu}_{m}{ }^{-}$ions $(\mathrm{m}$ $=1,2,3)$ with currents of 2, 0.6, $0.9 \mathrm{nA}$, respectively.

- Both $\mathrm{Si}_{m}{ }^{-}$and $\mathrm{Cu}_{m}{ }^{-}$ion beams could be focused on the analyzed sample surface into a $60 \mu \mathrm{m}$ spot providing current densities in the range of $0.04-0.08 \mathrm{~mA} / \mathrm{cm}^{2}$. The raster-scanned area was in the range $150 \times 150 \mu \mathrm{m}^{2}$.

- Instabilities in sputtered ion currents were in the range of $1 \%-2 \%$.

- The lifetime of the sputter ion source was about $300-400 \mathrm{~h}$ without both the reinstallation of the target and the reloading of the $\mathrm{CsCl}$ salt.

\section{$B$. The estimation of the oxygen coverage $\theta$ on the sputtered sample surfaces}

The yields of ions sputtered from the $\mathrm{Si}, \mathrm{Al}, \mathrm{InGaP}$, and GaAs samples with the $\mathrm{Si}_{m}{ }^{-}$and $\mathrm{Cu}_{m}{ }^{-}$ion beams as well as the ratio of these yields have been studied. To minimize the influence of the chemical effect (the dependence of the ion yields on the oxygen coverage $\theta$ of sample surfaces), the ion yields have been measured in conditions when $\theta$ of the sample surfaces sputtered with atomic and polyatomic projectiles were calculated from available data to be as low as possible (see below).

During experiments, the residual gas pressure did not exceed $10^{-8}$ Torr. Due to the fact that the ion gauge for the gas pressure measurement in the Cameca IMS- $4 \mathrm{f}$ instrument is placed near the throat of the pump, the pressure near the sample is at least an order of magnitude higher than that indicated by the gauge. So, it can be estimated as $\sim 10^{-7}$ Torr. Since there was no residual gas analyzer available, simple estimates were made for all studied "projectilesample" systems to see what would be the oxygen coverage $\theta$ of sample surfaces sputtered with the raster-scanned $\mathrm{Si}_{m}{ }^{-}$ and $\mathrm{Cu}_{m}{ }^{-}$ion beams if the assumed partial pressure of oxygen was exaggerated to $100 \%$. For these vacuum conditions, the surface arrival rate $\nu_{0}$ of oxygen atoms can be estimated by the known Hertz-Knudsen formula as $\sim 5$ $\times 10^{13} \mathrm{~cm}^{-2} \mathrm{~s}^{-1}$. For monoelement samples, the sputtering coefficients $K_{s}$ have been simulated with the TRIM computer $\operatorname{code}^{40}$ (the projectile incident angles needed for these simulations have been calculated with the SIMION 3D $\odot$ computer $\operatorname{code}^{39}$ ).

Assuming a sticking coefficient $\eta$ of 0.1 (Ref. 41) and using the surface atom removal rates $\beta K_{s} j_{0} / e(\beta \approx 0.16$ is the raster-scanned ratio), one can estimate the $\theta$ value as $\theta$ $=\eta \nu_{0} /\left(\beta K_{s} j_{0} / e+\eta \nu_{0}\right)$. The $\theta$ values are presented in Table $\mathrm{I}$. 
TABLE I. The oxygen coverage $\theta$ values estimated for the $\mathrm{Si}$ and $\mathrm{Al}$ sample surfaces sputtered with the raster-scanned $\mathrm{Si}_{m}{ }^{-}$and $\mathrm{Cu}_{m}{ }^{-}(m=1,2)$ ion beams.

\begin{tabular}{ccc}
\hline \hline Projectile & Target & $\theta$ \\
\hline $6.8 \mathrm{keV} \mathrm{Cu}^{-}$ & $\mathrm{Si}$ & 0.037 \\
$13.6 \mathrm{keV} \mathrm{Cu}_{2}{ }^{-}$ & $\mathrm{Si}$ & 0.052 \\
$6.8 \mathrm{keV} \mathrm{Cu}^{-}$ & $\mathrm{Al}$ & 0.027 \\
$13.6 \mathrm{keV} \mathrm{Cu}_{2}{ }^{-}$ & $\mathrm{Al}$ & 0.039 \\
$12 \mathrm{keV} \mathrm{Si}^{-}$ & $\mathrm{Si}$ & 0.013 \\
$12 \mathrm{keV} \mathrm{Si}_{2}{ }^{-}$ & $\mathrm{Si}$ & 0.013 \\
\hline \hline
\end{tabular}

These data should be considered as the very upper limit because the partial oxygen pressure was smaller than $\sim 10^{-7}$ Torr. Moreover, for diatomic ion bombardment, one can expect a further scaling down due to the increase of sputtering yields due to the nonadditive sputtering effect. Thus, in reality, the $\theta$ values as well as the difference in $\theta$ corresponding to the atomic and diatomic ion bombardments must be smaller than those presented in Table I.

\section{Nonadditive enhancement of a secondary ion emission}

It is known that the secondary ion emission from solids bombarded with $\mathrm{keV}$ atomic projectiles results mainly from linear cascade sputtering. ${ }^{42}$ Compared with atomic projectiles, polyatomic projectiles deposit a higher energy density in the impact region due to a spatial localization $\left(\sim 10^{-15} \mathrm{~cm}^{2}\right)$ and a temporal synchronization $\left(<10^{-14} \mathrm{~s}\right)$ of constituent atom collisions with a solid. According to the nonlinear cascade concept, ${ }^{43}$ a cluster ion impact produces nonlinear cascades which cause nonadditive enhancement in both sputtering ${ }^{4}$ and secondary ion yield. ${ }^{5,6}$ An enhancement factor $K_{m, 1}$, introduced to quantify nonadditive sputtering, is defined as

$$
K_{m, 1}=Y_{m} / m Y_{1},
$$

where $Y_{m}$ and $Y_{1}$ are yields of species of interest sputtered by $m$-atomic and atomic projectiles with the same velocity. Values of $K_{m, 1}$ depend on the projectile parameters, sample materials, and secondary species. The measurement of $K_{m, 1}$ can define more sensitive and/or accurate SIMS analytical conditions by choosing optimum combinations of these parameters. Due to its capability of producing a wide range of atomic and polyatomic ions, the sputter cluster ion source allows one to study the nonadditive effects for different projectile-sample systems.

As an example, a study of the nonadditive effect in secondary ion emission from the $\mathrm{Al}$ and $\mathrm{Si}$ samples bombarded with $6.8 \mathrm{keV} /$ atom $\mathrm{Cu}_{m}{ }^{-}$projectiles $(m=1,2)$ has been carried out. Before the start of the measurements the samples (purities 99.99\%) were cleaned (presputtered) by $\mathrm{Cu}^{-}$bombardment for $1 \mathrm{~h}$. Using Eq. (1), the values of $K_{2,1}$ were determined from secondary ion yields normalized according to the ratio of the corresponding primary ion currents. The values of $K_{2,1}$ for various sputtered ions are presented in Table II. These data show that, for the $\mathrm{Si}_{n}{ }^{-}$and $\mathrm{Al}_{n}{ }^{+}$ $(n=1-3)$ ion emissions, the $K_{2,1}$ factors increase with the increase of the number of atoms $n$ in the sputtered ion.
TABLE II. The values of the enhancement factor $K_{2,1}$ for secondary ion emissions from the $\mathrm{Si}$ and $\mathrm{Al}$ samples produced with the $6.8 \mathrm{keV} / \mathrm{atom} \mathrm{Cu}_{m}{ }^{-}$ $(m=1,2)$ projectiles.

\begin{tabular}{cccc}
\hline \hline Projectiles & Target & $\begin{array}{c}\text { Emitted } \\
\text { species }\end{array}$ & $\begin{array}{c}\text { Enhancement } \\
\text { factor } \\
K_{2,1}\end{array}$ \\
\hline $6.8 \mathrm{keV/atom}$ & $\mathrm{Si}$ & $\mathrm{Si}^{+}$ & 3.5 \\
$\mathrm{Cu}_{m}{ }^{-}(m=1,2)$ & & $\mathrm{Si}_{2}{ }^{+}$ & 4.1 \\
& & $\mathrm{Si}_{3}{ }^{+}$ & 6.1 \\
& $\mathrm{Al}$ & $\mathrm{Al}^{+}$ & 3.3 \\
& & $\mathrm{Al}_{2}{ }^{+}$ & 6.9 \\
& & $\mathrm{Al}_{3}{ }^{+}$ & 8.7 \\
\hline \hline
\end{tabular}

\section{Surface characterization of Si and InGaP samples}

Our previous studies ${ }^{5,6,44}$ have shown the different nonadditive effects in secondary cluster ion yields produced by polyatomic projectiles of heavy and light mass elements. Sputtering of the heavy (Ta), intermediate $(\mathrm{Nb})$, and light (Si) element samples with heavy $\mathrm{Au}_{m}{ }^{-}$projectiles $(m=2,3)$ results in a nonadditive enhancement that strongly increases with the number of atoms $n$ in the sputtered cluster ion and, for a given $n$, with the increase of the number $m \cdot{ }^{5,6}$ Results which follow a similar trend (see Sec. IV C) are observed during the bombardment of the light element samples (Al and $\mathrm{Si}$ ) with the intermediate mass element projectiles $\mathrm{Cu}_{m}{ }^{-}$ $(m=1,2)$. At the same time, sputtering of $\mathrm{Si}$ with the $\mathrm{Al}_{m}{ }^{-}$ $(m=1,2)$ projectiles ("light element target-light element projectile") has shown that the nonadditive enhancement for sputtered $\mathrm{Si}_{n}^{+}$ions $(n \leqslant 5)$ is not large. ${ }^{44}$ This distinction probably originates from the different energy densities deposited near the sample surface by heavy and light projectiles. One would expect that the energy density is varied with $E_{0} L^{-1}$, where $E_{0}$ and $L$ are the projectile energy and the mean penetration depth of projectile atoms. For $m$-atomic projectiles, the value of $L$ depends on the energy $E_{0} / m$ as well as the projectile and target atom masses. During the bombardment of silicon with $9 \mathrm{keV} /$ atom $\mathrm{Al}_{2}{ }^{-}$and $\mathrm{Au}_{2}{ }^{-}$ions, the value of $L$ is larger in the former case, so that for $\mathrm{Al}_{2}{ }^{-}$projectiles the cluster-to-target energy transfer process is shifted deeper into the bulk, resulting in a smaller nonadditive enhancement effect.

In this context, one can anticipate that the sputtering of heavy element samples with light element projectiles might enhance the ion emission due to the backscattering process which occurs when, in collisions with the heavy sample atoms, part of the light projectile constituent atoms recoil to the surface and deposit the additional energy in subsurface layers. Such a scenario, if proven to work, would be beneficial for SIMS applications with light element cluster ion beams. So, the following test was carried out.

$\mathrm{Si}^{-}$and $\mathrm{Si}_{2}{ }^{-}$ions with the energy of $12 \mathrm{keV}$ were used for the sputtering of the $\mathrm{Si}$ and $\mathrm{InGaP}$ samples. The specific question addressed here was a comparison of secondary ion yields from these samples produced with atomic and diatomic projectiles. The Si sample (light element) was doped with phosphorus to a concentration of $1.5 \times 10^{16} \mathrm{~cm}^{-3}$. The InGaP sample was selected because its composition represents heavy (In), intermediate (Ga), and light (P) atoms. The 
TABLE III. The normalized yields $Y_{m}(m=1,2)$ of secondary ions sputtered from the $\mathrm{Si}$ sample with the $12 \mathrm{keV} \mathrm{Si}_{m}{ }^{-}$projectiles $(m=1,2)$ and the ratio of $Y_{2} / Y_{1}$.

\begin{tabular}{cccc}
\hline \hline Species & $Y_{1}$ & $Y_{2}$ & $Y_{2} / Y_{1}$ \\
\hline${ }^{28} \mathrm{Si}^{+}$ & $4.0 \times 10^{-6}$ & $4.3 \times 10^{-6}$ & 1.1 \\
${ }^{28} \mathrm{Si}_{2}{ }^{+}$ & $2.6 \times 10^{-7}$ & $5.0 \times 10^{-7}$ & 1.9 \\
${ }^{28} \mathrm{Si}_{3}{ }^{+}$ & $3.6 \times 10^{-8}$ & $8.9 \times 10^{-8}$ & 2.5 \\
${ }^{28} \mathrm{Si}_{4}{ }^{+}$ & $1.7 \times 10^{-8}$ & $4.9 \times 10^{-8}$ & 2.9 \\
${ }^{28} \mathrm{Si}_{5}{ }^{+}$ & $5.6 \times 10^{-9}$ & $1.6 \times 10^{-8}$ & 2.8 \\
${ }^{12} \mathrm{C}^{+}$ & $1.4 \times 10^{-9}$ & $2.4 \times 10^{-9}$ & 1.7 \\
${ }^{16} \mathrm{O}^{+}$ & $3.0 \times 10^{-9}$ & $3.2 \times 10^{-9}$ & 1.1 \\
${ }^{23} \mathrm{Na}^{+}$ & $6.2 \times 10^{-9}$ & $2.1 \times 10^{-8}$ & 3.3 \\
${ }^{31} \mathrm{P}^{+}$ & $2.6 \times 10^{-9}$ & $5.2 \times 10^{-9}$ & 2.0 \\
${ }^{39} \mathrm{~K}^{+}$ & $1.0 \times 10^{-8}$ & $2.3 \times 10^{-8}$ & 2.3 \\
${ }^{28} \mathrm{Si}^{16} \mathrm{O}^{+}$ & $1.0 \times 10^{-8}$ & $1.3 \times 10^{-8}$ & 1.3 \\
\hline \hline
\end{tabular}

ion bombardment of InGaP initiates collision cascades involving a variety of atoms with different masses, and the study of secondary ion yields from this sample would reveal new regularities due to nonadditive sputtering.

Before the start of the measurements, the samples were cleaned (presputtered) for several hours by $\mathrm{Si}^{-}$ion bombardment. Mass spectra of positive ions sputtered from the samples were measured under the same experimental conditions. Various peaks were identified in the spectra corresponding to atomic and cluster ions of the main target element(s), ions of elemental impurities, as well as heterogeneous molecular ions. To compare the yields of the same secondary ions produced with the $\mathrm{Si}_{m}{ }^{-}$projectiles $(m$ $=1,2$ ), their peak intensities were normalized to the primary ion currents. Tables III and IV present the most intense normalized yields $Y_{m}(m=1,2)$ of ions sputtered from the $\mathrm{Si}$ and InGaP samples as well as their ratio $Y_{2} / Y_{1}$.

The general trend observed in these results is the enhancement of sputtered ion yields while changing from $\mathrm{Si}^{-}$to $\mathrm{Si}_{2}{ }^{-}$projectiles. The enhancement ratio $Y_{2} / Y_{1}$ depends on the sample and the sputtered species considered.

For the Si sample (Table III), the yields of atomic $\mathrm{Si}^{+}$

TABLE IV. The normalized yields $Y_{m}(m=1,2)$ of secondary ions sputtered from the InGaP sample with the $12 \mathrm{keV} \mathrm{Si}_{m}^{-}$projectiles $(m=1,2)$ and the ratio of $Y_{2} / Y_{1}$.

\begin{tabular}{cccc}
\hline \hline Species & $Y_{1}$ & $Y_{2}$ & $Y_{2} / Y_{1}$ \\
\hline${ }^{1} \mathrm{P}^{+}$ & $1.3 \times 10^{-8}$ & $4.8 \times 10^{-8}$ & 3.6 \\
${ }^{31} \mathrm{P}_{2}^{+}$ & $2.4 \times 10^{-9}$ & $1.0 \times 10^{-8}$ & 4.2 \\
${ }^{31} \mathrm{P}_{3}^{+}$ & $2.8 \times 10^{-9}$ & $4.0 \times 10^{-9}$ & 1.4 \\
${ }^{69} \mathrm{Ga}^{+}$ & $2.1 \times 10^{-6}$ & $1.6 \times 10^{-5}$ & 7.8 \\
${ }^{69} \mathrm{Ga}_{2}^{+}$ & $1.7 \times 10^{-7}$ & $2.5 \times 10^{-7}$ & 1.5 \\
${ }^{115} \mathrm{In}^{+}$ & $1.7 \times 10^{-5}$ & $5.7 \times 10^{-5}$ & 3.3 \\
${ }^{23} \mathrm{Na}^{+}$ & $1.4 \times 10^{-8}$ & $4.0 \times 10^{-6}$ & 285 \\
${ }^{28} \mathrm{Si}^{+}$ & $2.6 \times 10^{-8}$ & $3.0 \times 10^{-7}$ & 11.5 \\
${ }^{28} \mathrm{Si}_{2}^{+}$ & $1.6 \times 10^{-9}$ & $9.6 \times 10^{-9}$ & 6.0 \\
${ }^{28} \mathrm{Si}_{3}^{+}$ & $4.8 \times 10^{-10}$ & $9.6 \times 10^{-10}$ & 2.0 \\
${ }^{39} \mathrm{~K}^{+}$ & $1.2 \times 10^{-8}$ & $7.7 \times 10^{-7}$ & 66 \\
${ }^{69} \mathrm{Ga}^{31} \mathrm{P}^{+}$ & $7.6 \times 10^{-9}$ & $3.6 \times 10^{-8}$ & 4.7 \\
${ }^{115} \mathrm{In}^{31} \mathrm{P}^{+}$ & $3.4 \times 10^{-8}$ & $1.1 \times 10^{-7}$ & 3.3 \\
${ }^{115} \mathrm{In}^{69} \mathrm{Ga}^{+}$ & $1.2 \times 10^{-6}$ & $1.9 \times 10^{-6}$ & 1.6 \\
${ }^{28} \mathrm{Si}^{16} \mathrm{O}^{+}$ & $4.4 \times 10^{-10}$ & $2.1 \times 10^{-8}$ & 48 \\
\hline \hline
\end{tabular}

and cluster $\mathrm{Si}_{n}{ }^{+}(n=2-5)$ ions increase with factors ranging from 1.1 to 2.9 , which are in good agreement with previous data. ${ }^{44}$ The similar enhancement takes place for the yields of elemental $\left(\mathrm{C}^{+}, \mathrm{Na}^{+}, \mathrm{P}^{+}\right.$, and $\left.\mathrm{K}^{+}\right)$and molecular $\left(\mathrm{SiO}^{+}\right)$impurity ions. The largest enhancement (a factor of 3.3) is observed for $\mathrm{Na}^{+}$ions.

For the InGaP sample (Table IV), the enhancement of $Y_{2} / Y_{1}$ is more pronounced.

- The ion yields for the principal atomic components in the sample, i.e., ${ }^{115} \mathrm{In}^{+},{ }^{69} \mathrm{Ga}^{+}$, and $\mathrm{P}^{+}$ions, increase with factors of about 3.3, 7.8, and 3.6, respectively. Such ratios as $Y_{2} / Y_{1}$ are larger than that observed for the yield of the $\mathrm{Si}^{+}$ ions sputtered from silicon. At the same time, the yield of the ${ }^{69} \mathrm{Ga}_{2}{ }^{+}$ions increases only by a factor of 1.5 , while ratios $Y_{2} / Y_{1}$ for the $\mathrm{P}_{2}{ }^{+}$and $\mathrm{P}_{3}{ }^{+}$ions are about 4.2 and 1.4, respectively.

- The yields of the $\mathrm{Na}^{+}$and $\mathrm{K}^{+}$impurity ions are enhanced with factors of 285 and 66, respectively. This is similar to what we have observed for the Si sample, but the enhancement of these ion yields is much larger in the case of the InGaP sample, demonstrating that the cluster ion bombardment could increase the sensitivity of SIMS in trace analysis applications.

- The ratios $Y_{2} / Y_{1}$ of the $\mathrm{Si}^{+}, \mathrm{Si}_{2}{ }^{+}$, and $\mathrm{Si}_{3}{ }^{+}$ions are of 11.5, 6 , and 2, respectively. Thus, for the $\mathrm{Si}_{n}{ }^{+}$ions sputtered from the $\mathrm{Si}$ and $\mathrm{InGaP}$ samples, the dependence of $Y_{2} / Y_{1}$ on the number $n$ has a different trend. For the Si sample (when $\mathrm{Si}_{n}{ }^{+}$ions originate from the sample matter), $Y_{2} / Y_{1}$ increases with $n$. On the contrary, for the InGaP sample (when $\mathrm{Si}_{n}{ }^{+}$ions originate from the implanted $\mathrm{Si}$ atoms), $Y_{2} / Y_{1}$ decreases with $n$. This difference shows that the emission of clusters consisting of implanted atoms depends on the density of neighboring implanted atoms located near the emission point. For the InGaP sample the $Y_{2} / Y_{1}$ values for sputtered $\mathrm{Si}^{+}$and $\mathrm{Si}_{2}{ }^{+}$ions are larger than those observed for the $\mathrm{Si}$ sample, once again underlining the advantages of using cluster projectiles in SIMS for an analysis of impurities, such as, in this case, implants.

- The yields of the $\mathrm{SiO}^{+}$ions strongly increase compared to those observed for the Si sample. Taking into account that (i) the oxygen coverage values estimated for the Si sample bombarded with the $\mathrm{Si}^{-}$and $\mathrm{Si}_{2}{ }^{-}$projectiles are the same (see Table I) and (ii) the $\mathrm{O}^{+}$yields from both samples are small and do not change in going from atomic to diatomic projectiles (see Tables III and IV), one can suppose that the increase of the $\mathrm{SiO}^{+}$yield is rather connected with an increase in implanted $\mathrm{Si}$ atoms rather than with an increase of adsorbed oxygen atoms on the sputtered InGaP surface.

- Yields of the $\mathrm{GaP}^{+}, \mathrm{InP}^{+}$, and $\mathrm{InGa}^{+}$heterogeneous ions are increased with factors of 4.7, 3.3, and 1.6, respectively.

In summary, the ion yield enhancement due to the polyatomic ion bombardment was observed for both samples, but this process is much stronger for the $\mathrm{InGaP}$ than for $\mathrm{Si}$. The general cause of this enhancement can be attributed to the development of nonlinear collision cascades. However, the distinction in the enhancements for these samples cannot be explained just from this single assumption, rather it originates from differences in the evolution of the collision cas- 
cades in $\mathrm{Si}$ and $\mathrm{InGaP} .{ }^{45}$ Such a distinction can be connected with the backscattering process mentioned above, which opens an additional channel for the energy transfer from projectile atoms to those in the subsurface layers of the sample. For light atomic projectiles, the contribution of backscattering to the sputter yield for light species chemisorbed on a substrate of heavier atoms has been studied theoretically by Winters and Sigmund. ${ }^{46}$ The efficiency of backscattering should increase with both decreasing energy $E_{0}$ and increasing atomic weight of the target atom. For a given energy $E_{0}$, the efficiency of backscattering will be higher for atoms of the polyatomic projectile than for the atomic projectile because of the partitioning of $E_{0}$. In this context, the sample made from a heavy weight element can be considered as a "diffusion mirror" concentrating the energy deposited by the backscattered light atoms of polyatomic projectiles in the subsurface layers of the sample. Thus, compared with the bombardment of the light element sample, the bombardment of the heavy element sample with the light element polyatomic projectiles can lead to an increased density of energy deposited in the subsurface layers and, hence, to a larger nonadditive enhancement of secondary ion emission.

It should be noted that collision cascades developing in the subsurface layers of the InGaP sample can involve a variety of the heavy (In), intermediate (Ga), and light (P) weight atoms as well as light projectile atoms $(\mathrm{Si})$ and low atomic number impurities. During these processes, energetic $\mathrm{Si}$ atoms can more efficiently transfer energy to lighter partners in the collision, such as phosphorus, silicon, and impurity atoms, and this can lead to a stronger enhancement in the emission yields of these ions.

\section{E. Depth profiling measurements}

For several years now, SIMS depth profiling evolved to encompass the use of low-energy ions $(<250 \mathrm{eV})$ to achieve nanometer-scale depth resolution as a result of a reduced ion range. However, the depth resolution cannot be improved further without a significant loss of sensitivity due to the physical limits of sputtering by atomic ions (or light diatomic ones, such as $\mathrm{O}_{2}^{+}$). This difficulty might be avoided by using cluster ion projectiles of heavy and intermediate weight elements, taking advantage of the following known features of polyatomic ion bombardment: (1) enhanced depth resolution through partitioning of the kinetic energy of polyatomic projectiles (for a given projectile energy, a heavier cluster constituent atom has a shorter ion range compared with an atomic ion); (2) the narrow energy spread of the primary ions (this depends on the nature of the source); (3) enhanced secondary ion emission through nonlinear sputtering effects; and (4) high surface erosion rates for low total primary ion currents. Optimization of all these should result in the improvement of the depth resolution. However, the question of how effective cluster ions of heavy and intermediate weight elements might be for SIMS depth profiling still remains open.

Currently, the generation of intense and well focused low energy ion beams $\left(E_{0}<500 \mathrm{eV}\right)$ in commercial SIMS instruments can be achieved using a special technique such as the floating low energy ion gun (FLIG) developed by Dowsett $e t$

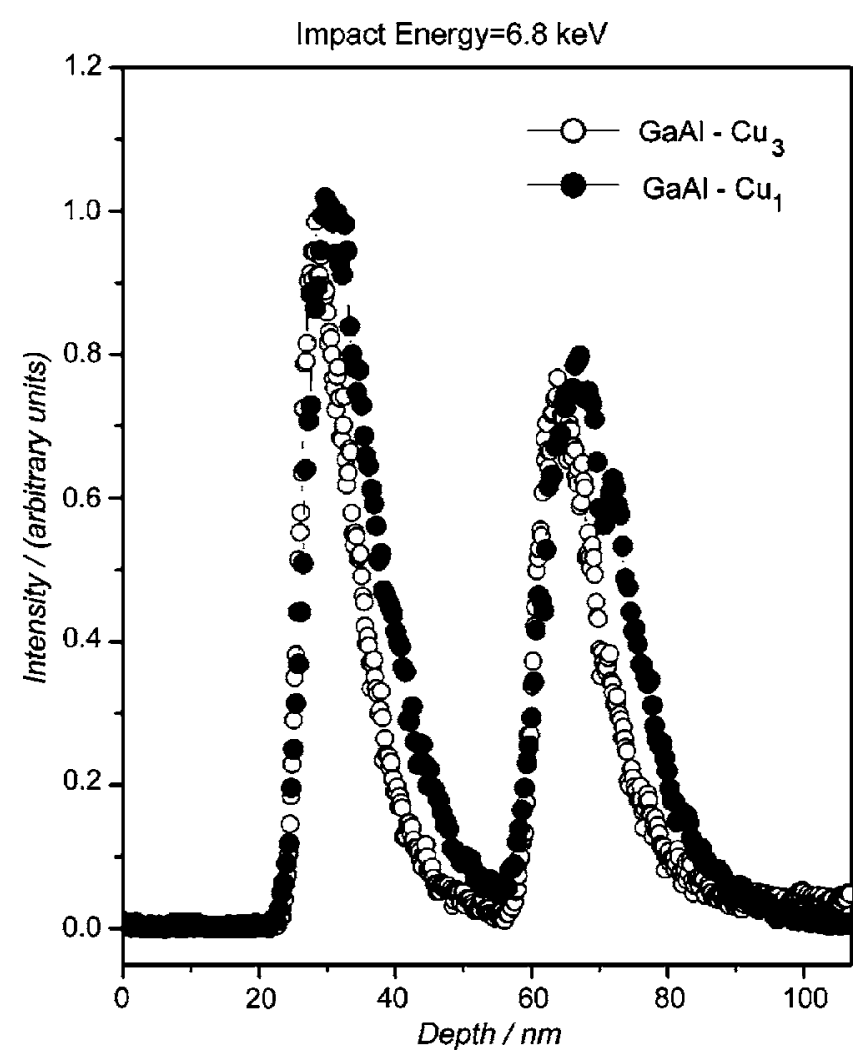

FIG. 3. Depth profiling of the delta AlAs layers incorporated in the GaAs sample measured with the $6.8 \mathrm{keV}$ atomic $\mathrm{Cu}^{-}$and cluster $\mathrm{Cu}_{3}{ }^{-}$projectiles. The sample contains two $\sigma$-AlAs layers. The distance between $\sigma$-AlAs layers as well as the depth of the uppermost layer was $32 \mathrm{~nm}$.

$a l .{ }^{47}$ However, the Cameca IMS-4f instrument does not permit the use of low energy negatively charged primary ions when positive secondary ions are to be detected (see Sec. III). Moreover, the primary mass separator of this instrument is not capable of deflecting/resolving heavy molecular ions. Therefore, the objective of this test was to study the SIMS depth profiling using the atomic and cluster projectiles of the intermediate weight elements with the minimal kinetic energy.

In an attempt to do this, the raster-scanned $6.8 \mathrm{keV} \mathrm{Cu}^{-}$ and $\mathrm{Cu}_{3}{ }^{-}$ion beams with the currents of 2 and $0.9 \mathrm{nA}$ (the raster-scanned parameters are presented in Sec. IV A) were used for SIMS depth profiling of a model sample prepared by means of molecular beam epitaxy. This consists of an epitaxial layer of GaAs delta doped with two AlAs marker layers.

The distance between $\sigma$-AlAs layers as well as the thickness of the uppermost layer were $32 \mathrm{~nm}$. The depth profiles were obtained for the ${ }^{27} \mathrm{Al}^{+}$ions emitted from the crater bottom with a diameter $60 \mu \mathrm{m}$. These results are presented in Fig. 3. They show that, compared with $6.8 \mathrm{keV} /$ atom $\mathrm{Cu}^{-}$ projectiles, the use of $2.26 \mathrm{keV} /$ atom $\mathrm{Cu}_{3}{ }^{-}$projectiles improves the depth resolution [according to the full width at half maximum (FWHM) definition, the depth resolution is increased by a factor of 1.3]. However, this improvement is not substantial. To understand it, we have compared these results with the results of Ref. 20, where, for the same $\mathrm{GaAs} / \sigma$-AlAs $/ \mathrm{GaAs} / \cdots / \mathrm{GaAs}$ sample, depth profiling measurements have been performed on the Cameca IMS-4f 
instrument with the standard duoplasmatron ion source and the $8 \mathrm{keV} \mathrm{O}_{m}{ }^{+}(m=1-4)$ ions have been used as projectiles. In this case, the raster-scanned parameters of the $\mathrm{O}_{m}{ }^{+}$ion beams were better (the beams were focused into a spot with a diameter of $20 \mu \mathrm{m}$ and scanned over a $250 \times 250 \mu \mathrm{m}^{2}$ area on the sample surface). However, compared with the $8 \mathrm{keV} /$ atom $\mathrm{O}^{+}$projectiles, the use of the $2.66 \mathrm{keV} /$ atom $\mathrm{O}_{3}{ }^{+}$ projectiles improved the depth resolution only by a factor of 1.4 , which is very close to our results. Thus, a comparison of our results with the results of Ref. 20 shows that the nonsubstantial improvement in the depth resolution is rather connected with the used projectile energy range than with the used raster-scanned parameters of ion beams.

\section{DISCUSSION}

To address the problem of the enhancement of analytical capabilities of the existing dynamic SIMS equipment such as a Cameca magnetic sector (IMS-xf series) and quadrupole (4500/4550 series) instruments, a sputter cluster ion source has been developed. This device was tested on the Cameca IMS-4f magnetic sector instrument. A number of experiments have been carried out in the following: (1) studies of nonadditive enhancements in secondary ions emission, (2) surface characterization of Si and InGaP samples, and (3) depth profiling measurements. Experimental results indicate that the use of polyatomic ions as projectiles for sputtering in inorganic SIMS, instead of atomic ones, has some advantages. Among these, an enhanced sputter yield is demonstrated for both atomic and polyatomic secondary ions, which altogether translates into improved detection limits. Another advantage that is demonstrated is improved depth resolution. Nevertheless, it remains to be seen how the depth resolution and sensitivity compare with those of conventional probes such as the $\mathrm{O}_{2}^{+}$ions with energies below $1 \mathrm{keV}$.

The test results show that the developed design of the cluster ion source can be used as a simple and cost-saving solution for an upgrade of the expensive mainframe of SIMS instrumentation. From the perspective of developing a fundamental understanding, however, it is apparent that systematic studies of sputtering with polyatomic ions should be continued, for example, by using different projectile-target combinations, especially for different relationships between atomic weights of elements that form the projectile and the target and for different ranges of impact energies, especially for those in the low range approaching the sputtering threshold. In connection with this, a low energy column of the type used on Cameca 4500/4550 quadrupole instruments (FLIG $\left.{ }^{\mathrm{TM}}\right)$ is being developed with a matched sputter cluster source. This will allow systematic studies of nonadditive sputtering of solids with the low energy heavy cluster projectiles for conditions that are not allowed with the present source.

\section{ACKNOWLEDGMENTS}

The authors are grateful to the mechanics G. P. Ryntsev (Uzbekistan), R. Corremans (Belgium), and J.-P. Huysmans
(Belgium) for their help in producing the cluster ion source design. They also acknowledge financial support from NATO (SfP Project No. 97-1929), the Belgian Federal Services for Scientific, Technical, and Cultural Affairs (DWTC/SSTC) of the Prime Minister's Office through IUAP-IV (Conv. P4/10), and EPSRC (Project No. GR/S78919/01). One of the authors (I.V.V.) acknowledges support from the U.S. Department of Energy, BES-Materials Sciences under Contract No. W-31109-ENG-38. Another author (M.G.D.) acknowledges support from Cameca $\mathrm{GmbH}$.

${ }^{1}$ M. G. Dowsett, Appl. Surf. Sci. 203-204, 5 (2003).

${ }^{2}$ P. Bertrand, A. Delcourt, and B. J. Garrison, Appl. Surf. Sci. 203-204, 160 (2003).

${ }^{3}$ C. M. John and R. W. Odom, Int. J. Mass Spectrom. Ion Process. 161, 47 (1997).

${ }^{4}$ Y. Le Beyec, Int. J. Mass Spectrom. Ion Process. 174, 101 (1998).

${ }^{5}$ S. F. Belykh, B. Habets, U. Kh. Rasulev, A. V. Samartsev, L. V. Stroev, and I. V. Veryovkin, Nucl. Instrum. Methods Phys. Res. B 164-165, 809 (2000).

${ }^{6}$ S. F. Belykh, U. Kh. Rasulev, A. V. Samartsev, L. V. Stroev, and A. V. Zinoviev, Vacuum 56, 257 (2000).

${ }^{7}$ G. Gillen, L. King, and F. Chmara, J. Vac. Sci. Technol. A 17, 845 (1999).

${ }^{8}$ N. Toyoda, J. Matsuo, T. Aoki, I. Yamada, and D. B. Fenner, Appl. Surf. Sci. 203-204, 214 (2003).

${ }^{9}$ S. C. C. Wong, R. Hill, P. Blenkinsopp, N. P. Lockyer, and J. C. Vickerman, Appl. Surf. Sci. 203-204, 219 (2003).

${ }^{10}$ I. Yamada, J. Matsuo, Z. Insepov, T. Aoki, T. Seki, and N. Toyoda, Nucl. Instrum. Methods Phys. Res. B 164-165, 809 (2000).

${ }^{11}$ J. A. Townes, A. K. White, E. N. Wiggins, K. D. Krantzman, B. J. Garrison, and N. Winograd, J. Phys. Chem. A 103, 4587 (1999).

${ }^{12}$ P. Bertrand, A. Delcorte, B. J. Garrison, Appl. Surf. Sci. 203-204, 160 (2003).

${ }^{13}$ A. Delcorte, P. Bertrand, and B. J. Garrison, Appl. Surf. Sci. 203-204, 166 (2003).

${ }^{14}$ R. Žaric, B. Pearson, K. D. Krantzman, and B. J. Garrison, Int. J. Mass Spectrom. Ion Process. 174, 155 (1998).

${ }^{15}$ R. Žaric, B. Pearson, K. D. Krantzman, and B. J. Garrison, in Secondary Ion Mass Spectrometry SIMS XI, edited by G. Gillen, R. Lareau, J. Bennett, and F. Stevie (John Wiley \& Sons Ltd., Orlando, 1998), p. 601.

${ }^{16}$ M. Medvedeva, I. Wojciechowski, and B. J. Garrison, Surf. Sci. 505, 34 (2002).

${ }^{17}$ A. D. Appelhans and J. E. Delmore, Anal. Chem. 61, 1087 (1989).

${ }^{18}$ K. Iltgen, C. Bendel, A. Benninghoven, and E. Niehuis, J. Vac. Sci. Technol. A 15, 460 (1997).

${ }^{19}$ H. Yamazaki and Y. Mitani, Nucl. Instrum. Methods Phys. Res. B 124, 91 (1997).

${ }^{20}$ B. Y. Ber, A. P. Kovarsky, D. Y. Kazantsev, Y. Y. Trushin, E. E. Zhurkin, A. A. Schmidt, and S. F. Belykh, Tech. Phys. Lett. 30, 836 (2004).

${ }^{21}$ N. Devies, D. E. Weibel, P. Blenkinsopp, N. Lockyer, R. Hill, and J. C. Vickerman, Appl. Surf. Sci. 203-204, 223 (2003).

${ }^{22}$ R. Hill and P. W. M. Blenkinsopp, Appl. Surf. Sci. 231-232, 936 (2004).

${ }^{23}$ R. Hill, P. W. M. Blenkinsopp, A. Barber, and C. Everest, Appl. Surf. Sci. 252, 7304 (2006).

${ }^{24}$ D. Touboul, F. Kollmer, E. Niehuis, A. Brunelle, and O. Laprévote, J. Am. Soc. Mass Spectrom. 16, 1608 (2005).

${ }^{25}$ J. S. Fletcher, N. P. Lockyer, and J. C. Vickerman, Surf. Interface Anal. 38, 1393 (2006)

${ }^{26}$ A. Kh. Ajukhanov and V. N. Chernenko, Prib. Tekh. Eksp. 2, 150 (1972) (in Russian).

${ }^{27}$ R. Middleton and C. T. Adams, Nucl. Phys. 118, 329 (1974).

${ }^{28}$ G. D. Alton, Rev. Sci. Instrum. 63, 2450 (1992).

${ }^{29}$ R. Hopfl et al., Rev. Sci. Instrum. 63, 2469 (1992).

${ }^{30}$ S. Houzhi, Z. Weizhong, Z. Jinhua, D. Guangtian, and Z. Tiaorong, Rev. Sci. Instrum. 63, 2472 (1992)

${ }^{31}$ X. M. Wang, X. M. Lu, L. Shao, J. R. Liu, and W. K. Chu, Nucl. Instrum. Methods Phys. Res. B 196, 198 (2002).

${ }^{32}$ S. F. Belykh, R. N. Evtukhov, Ju. N. Lysenko, and U. Kh. Rasulev, Rev. Sci. Instrum. 63, 2458 (1992).

${ }^{33}$ G. Gillen, L. King, B. Freibaum, R. Lareau, J. Bennett, and F. Chmara, J. Vac. Sci. Technol. A 19, 568 (2001).

${ }^{34}$ G. Gillen and A. Fahey, Appl. Surf. Sci. 203-204, 209 (2003). 
${ }^{35}$ Development of a negative cluster ion source for mass spectrometry of secondary ions and secondary neutrals. NATO SfP Program, Project No. 97-1929.

${ }^{36}$ R. Middleton, Nucl. Instrum. Methods 144, 373 (1977).

${ }^{37}$ S. F. Belykh and R. N. Evtukhov, Prib. Tekh. Eksp. 2, 191 (1982) (in Russian).

${ }^{38}$ I. V. Veryovkin, W. F. Galaway, and M. J. Pellin, Nucl. Instrum. Methods Phys. Res. A 519, 353 (2004).

${ }^{39}$ D. A. Dahl, Int. J. Mass. Spectrom. 200, 3 (2000).

${ }^{40}$ J. P. Ziegler, J. P. Biersack, and U. Littmark, The Stopping and Range of Ions in Solids (Pergamon, Oxford, 1985), Vol. 1.

${ }^{41}$ T. Yano, T. Ooie, M. Yoneda, M. Katsumura, T. Hino, and T. Araki, J. Jpn.
Inst. Met. 61, 71 (1997)

${ }^{42}$ M. L. Yu, in Sputtering by Particle Bombardment III, edited by R. Behrisch (Springer, Berlin, 1991), p. 91.

${ }^{43}$ P. Sigmund and C. Claussen, J. Appl. Phys. 52, 990 (1981).

${ }^{44}$ S. F. Belykh, A. P. Kovarsky, V. V. Palitsin, A. Adriaens, and F. Adams, Int. J. Mass. Spectrom. 209, 141 (2001).

${ }^{45}$ M. G. Dowsett, Ph.D. thesis, CNAA, 1977.

${ }^{46}$ H. F. Winters and P. Sigmund, J. Appl. Phys. 45, 4760 (1974).

${ }^{47}$ M. G. Dowsett, N. S. Smith, R. Bridgeland, D. Richards, A. C. Lovejoy, and P. Pedrick, in Secondary ion Mass Spectrometry SIMS X, edited by A. Benninghoven (Wiley, New York, 1997), p. 367. 